\title{
Paramedic Perception of Endotracheal Intubation Performance: an Examination of Irish Advanced Paramedic Demographics, Characteristics, Confidence and Perceived Barriers to the Performance of Advanced Airway Management.
}

\section{Alan Horan}

Cork University Hospital, HSE South https://orcid.org/0000-0002-1369-6997

\section{Siobhán Masterson}

HSE National Ambulance Service, National University of Ireland Galway

\section{Cathal O'Donnell}

HSE National Ambulance Service, University of Limerick

David Hennelly ( $\nabla$ david.hennelly@hse.ie )

Clinical Directorate, National Ambulance Service https://orcid.org/0000-0001-5297-8244

Original research

Keywords: Paramedic, Advanced Paramedic, Intubation, Airway Management, Education

Posted Date: October 11th, 2021

DOl: https://doi.org/10.21203/rs.3.rs-956218/v1

License: (9) This work is licensed under a Creative Commons Attribution 4.0 International License. Read Full License 


\section{Abstract}

\section{Background}

Much research has occurred internationally with regard to the prehospital ETI (endotracheal intubation), however to date little is known of Paramedics perception of the procedure. In order to gain insight into procedural perception Irish Advanced Paramedics (AP) were invited to participate in an electronic survey. This survey attempted to gather information surrounding AP's experience of education for and performance of ETI, to identify procedural barriers and gain insight in to the continuing developmental needs of AP's to maintain confidence and competence in ETI performance.

\section{Methods}

An online questionnaire was created and AP's employed by the National Ambulance Service and the Dublin Fire Brigade, were invited to participate. The objective was to measure the characteristics, attitudes and perceived barriers to ETI by AP's in Ireland. Participants were asked to categorise their personal characteristics of ETI (frequency, techniques, barriers) through a series of 36 structured questions and answers.

\section{Results}

Of the 524 AP surveyed the response rate was $27 \%(n=140) 77.9 \%$ of respondents perform ETI 10 times or less per year. $26.6 \%$ of respondents maintain a personal airway management log book. $97.8 \%$ of respondents reported ETI as being an important AP skill. Most felt confident at performing the procedure but felt it was of moderate difficulty.

There was a lack of consensus on the definition of a failed intubation attempt. Initial supervised intubation practice in Hospital or the clinical skill lab was felt to be very important. Most respondents felt that there should be a minimum number of intubations performed by a paramedic each year, and that if this number was not achieved in the pre-hospital setting in-hospital practice should be an available alternative.

\section{Conclusion}

ETI is perceived to be an important skill by Paramedics. In practice there is wide variances in standards of data reporting, continuing assessment and competency assurance in ETI. A hybrid approach of individuals maintaining an airway portfolio which encompasses a clinical airway logbook, self-directed airway simulation with periodic senior peer appraisal and in-hospital clinical feedback may be the best approach for Paramedics with limited pre-hospital advanced airway management opportunities.

\section{Background}


For patients who have a clinical indication for pre-hospital endotracheal intubation (ETI) it is recommended that it should be performed by paramedics or physicians who have adequate experience and competence. These clinicians should be supported by a robust clinical governance structure and a well-governed system of continual training, education and competency assessment [1].

Pre-hospital airway management is a complex process and involves the operator to complete multiple separate cognitive and manual tasks simultaneously [2]. In a large retrospective registry review in King County, USA 1.4\% of Emergency Medical Service (EMS) activations required an ETI [3], therefore prehospital ETI can be classified as a High Acuity Low Occurrence (HALO) procedure. International evidence and consensus statements have identified low numbers of supervised in-hospital ETI practice and low exposure to pre-hospital ETI as barriers to gaining and maintaining successful ETI competency $[4,5,6]$.

Paramedic intubation experience has been reported to increase ETI procedural success [5] while procedural confidence is multifactorial [7]. Much debate has occurred regarding the base profession of the individual performing ETI [8]. A retrospective observational study of ETI performed by a Pre-hospital Critical Care team which consisted of a Paramedic and a Physician described no difference in actual procedural ETI success rates between the professions [9].

Our research examines the demographics, airway management characteristics, confidence and perceived barriers to the performance of ETI. This research also attempts to gain insight in to the continuing developmental needs to maintain confidence and competence in ETI performance of Advanced Paramedics (APs) in the Irish healthcare setting.

\section{Methods}

EMS in Ireland is provided by the National Ambulance Service and the Dublin Fire Brigade. The EMS system serves a population of approximately 4.7 million with a population density of $70 / \mathrm{km}$, with $44 \%$ of the population concentrated in Dublin, Irelands capital city [10]. Since 2004 a two-tier EMS response is employed in Ireland with 524 of the total 2500 approximate EMS staff having completed the Advanced Paramedic program which designates them as Advanced Life Support (ALS) providers. Registered AP's are deployed across multiple platforms including emergency ambulance, rapid response vehicle and rotary wing aircraft. They are permitted to perform ETI without pharmacological assistance in accordance to an advanced airway management clinical practice guideline [11]. As a result of this the majority of patients who are intubated in the pre-hospital setting will either be in respiratory or cardiac arrest.

Exposure to ETI is likely to vary significantly due to frequency of patient interaction and density of population served; this also has an impact on opportunities for on-going training and assessment of competence.

An online questionnaire was created and APs employed by each organisation were invited to participate. The objective of the survey was to describe the characteristics, attitudes and perceived barriers to ETI by APs in Ireland. 
Invitation was made available by means of a quick response (QR) code and web link displayed on the survey advertisement which was attached to the clinical notice board of each EMS station in the country. In addition the education manager responsible for each geographic region of the country was notified of the survey and requested to forward on to local staff email lists. The survey was hosted by Google@ and was live for two months with a repeat email reminder sent midway through the survey period.

Consent was obtained for the study through the University Hospital Limerick, Ireland research ethics committee and approval to conduct the survey was also approved by the National Ambulance Service research committee.

Participants were asked to categorize their personal characteristics of ETI (frequency, techniques, barriers) through a series of structured questions and answers. Participants who did not keep a record of previous ETI attempts were asked to estimate.

Ten-point Likert scales were employed to measure confidence and attitudes in performing airwaymanagement procedures (e.g. $1=$ not confident $10=$ very confident $/ 1=$ not difficult $10=$ very difficult)

\section{Results}

\section{Demographics}

The survey response rate was $27 \%(n=140) .42 \%$ of respondents had less than 5 years' experience as an AP. $35 \%$ had between 5 and 10 years of experience and $22.9 \%$ had greater than 10 years' experience. $45.7 \%$ reported that they are primarily deployed into a mixed urban and rural environment while $40.6 \%$ mainly worked in an urban setting, $13 \%$ stated they worked in a mainly rural setting.

\section{Characteristics}

$28.6 \%$ of respondents maintained a personal airway logbook.

$77.9 \%$ performed ETI 10 times or less per year while $2.9 \%$ of respondents reported 0 attempts per year, of the remaining respondents $3.6 \%$ reported attempting between 20 and 30 while $2.9 \%$ reported between 30 and 40 .

$55.4 \%$ reported that their attempts were successful between $75 \%$ and $99 \%$ of the time while $20 \%$ reported success between 50 to $75 \%$ of the time. $3.7 \%$ of respondents reported that $100 \%$ of their intubation attempts resulted ETI being used to successfully ventilate the patient,

$95 \%$ of respondents reported that their most commonly used method following a failure perform ETI was a supraglotic airway device (61.2\% King LT@, 33.1\% Laryngeal Mask Airway (LMA), 1.4\% iGel@) while $2.9 \%$ reported Oropharyngeal Airway (OPA) and 1.4\% Nasopharyngeal Airway (NPA) being the most commonly used rescue device. 
$90 \%$ of respondents reported that waveform capnography was always available to them, $100 \%$ indicated that they felt having end tidal capnography was useful during ETI.

With regard to endotracheal tube introducers respondents were asked about the use of bougies and stylets. $32.4 \%$ of respondents always utilised a bougie, $25.2 \%$ used a bougie only they anticipate a difficult ETI, $15.8 \%$ use a bougie if they can get an adequate view of the vocal cords but cannot pass the endotracheal tube (ETT) through the cords on the first attempt while 19.4 report that they have never used a bougie.

$84.1 \%$ of respondents have never used a stylet, $10 \%$ state they utilise a stylet if they anticipate difficulty, $2.9 \%$ of AP's reported that they use a stylet for every ETI while $2.9 \%$ use a stylet if they can obtain an adequate view of the vocal cords but cannot pass the ETT on the first attempt.

With regard to the definition of a failed ETI attempt participants were proposed 5 different definitions. The results were as follows:

- $45.7 \%$ reported that this was defined as when the operator is unable to pass an ETT and/or bougie through the vocal cords resulting in an alternative airway device being used.

- $22.9 \%$ of respondents indicated that a failed intubation attempt was an intubation attempt which resulted in confirmation that the ETT was in the oesophagus.

- $16.4 \%$ of respondents felt it was a failure to pass an ETT/bougie through the vocal cords which resulted in changing the position of the operator and/or patient.

- $13.6 \%$ felt it was a failure to view the vocal cords at laryngoscopy which resulted in changing the position of the operator and/or patient.

- $1.4 \%$ felt it was a failure to pass an ETT through the cords resulting in the inability to ventilate and oxygenate the patient requiring a needle cricothyroidotomy

With regard to post ETI documentation when given the option to select multiple answers $97 \%$ reported documenting the end tidal $\mathrm{CO} 2$ value and presence of a capnographic waveform, $66 \%$ documented the number of attempts at ETI, $24 \%$ documented the Cormac \& Lehane grading scale, $67 \%$ recorded the length of the ETT at the teeth while $27 \%$ recorded the length of the ETT at the lips, $89 \%$ reported that they routinely recorded the presence of bilateral breath sounds to auscultation.

\section{Attitudes \& Barriers}

$98.6 \%$ of respondents felt that ETI should be part of AP scope of practice. $40 \%$ of respondents felt that the skill of ETI was very important

The majority of the respondents considered the skill to be at least moderately difficult.

The majority of the respondents were at least moderately confident at performing ETI. 
When given the option to select multiple answers regarding the most common barriers to successful ETI $65 \%$ reported anatomical variability, $56 \%$ reported an inability to adequately position the patient, $52.1 \%$ stated the most common barrier was the presence of a soiled airway, $35 \%$ reported deranged anatomy while $5 \%$ reported age of the patient.

$72.9 \%$ of respondents felt that the skill of laryngoscopy for foreign body removal was very important. The majority of the respondents considered the skill to be at least moderately difficult. In relation to the supporting role of EMS colleagues during ETI, 58.6\% of respondents felt that other members of the EMS team were adequately trained to assist them.

\section{Education and CPC}

$60 \%$ of respondents have attended airway management continuing education programs since their initial training with annual in-service resuscitation update being the most common form of airway education.

Respondents were questioned regarding the quality of their educational experience to prepare them for ETI. The majority of the respondents reported that initial supervised in hospital ETI experience was important.

$71 \%$ of respondents felt that on-going skill maintenance in ETI was very important, yet there was no consensus amongst the respondents with regard to their ongoing skill maintenance $\mathrm{CPC} /$ experience.

$77.9 \%$ of respondents felt that they should be required to perform a predetermined minimum number of ETI per year.

Survey respondents were given multiple options to suggest how competency should be maintained if unable to meet the minimum requirement, it was possible to select more than option. $79.5 \%$ felt that inhospital supervision was the best mechanism for ensuring competency, $19.7 \%$ felt that pre-hospital supervised practice was best, $52 \%$ felt simulation on mannequins was best, $36.2 \%$ felt simulation in a cadaver lab was best while $21.3 \%$ felt rotation to a busier geographic setting was the best approach.

\section{Discussion}

It was apparent amongst respondents that no standard definition of a failed ETI attempt exists and 33\% did not routinely document the number of intubation attempts. This information has previously been identified as paramount to airway reporting [12]. The recent introduction of Electronic Patient Care Reporting (EPCR) in the prehospital setting in Ireland creates opportunities to prompt users to populate key procedural information on the EPCR and to generate an individual airway management log automatically.

It has previously been recommended that EMS should monitor Paramedic ETI experience and seek ways to increase it [4]. The Consensus statement by the UK College of Paramedics has identified 24 ETI per

year as a requirement for competence. The majority of our respondents indicated they perform ETI on 10 
or less occasions per year. Prekker et al have reported how a two tier EMS response which preferentially dispatches Paramedic teams with an advanced airway skillset to those patients most likely to require ETI can maximize exposure and improve performance when supported by a robust governance structure [3].

Independent practice combined with periodic feedback has previously been demonstrated to be of value [13]. Survey respondents favored in-hospital supervision as the best mechanism for maintaining competency in the event of inadequate exposure, therefore strong links between EMS and local Anaesthesiology departments are important.

Regular supervised ETI experience can increase exposure and result in a first pass success rate of $95 \%$ and adequate management of failed intubation attempts [14].

However, this form of continuing education is associated with a financial cost due to clinical service maintenance throughout training [15]. Allied healthcare professionals have demonstrated the benefits of self-directed simulated practice that is visually recorded and self-reviewed [16]. This model could be further developed through the introduction of a remote review process by senior peers facilitated by an internet based leaning hub.

This survey was performed prior to the covid-19 pandemic. At the commencement of the pandemic airway management techniques during resuscitation were subject to scrutiny. During this time ETI was suspended from Irish pre-hospital practice in favor of supraglotic airway device insertion. This strategy was intended to limit occupational exposure and decrease disease transmission associated with prehospital resuscitation efforts. With a greater than 12 month hiatus from ETI exposure this has resulted in a further barrier in the form of a loss in exposure to a procedure which already is a low occurrence for many individual clinicians.

\section{Conclusion}

ETI is perceived to be an important ALS Paramedic skill. There is currently limited international consensus regarding the continuing assessment of competence in ETI for Paramedics.

A hybrid approach of individuals maintaining an airway portfolio which encompasses a clinical airway logbook, self-directed airway simulation with periodic senior peer appraisal and in-hospital clinical feedback may be the best approach for a system of advanced life support (ALS) Paramedics who are faced with a wide variation in clinical exposure.

EMS organizations should seek ways to moderate the number of ALS Paramedics who are required to carry out HALO Procedures such as prehospital ETI, striving for a high quality rather than a high quantity approach with comprehensive continuing education and clinical governance structures, dispatching them preferentially to critically ill and injured patients in order to maximize procedural opportunity and success. Furthermore for EMS systems which experienced a reduction in exposure for individual clinicians to 
maintain ongoing exposure to ETI during the Covid-19 pandemic there now exists an opportunity to reexamine how ETI competence is maintained.

Further research in the form of a national prehospital airway audit is required to gain better insight into the characteristics of airway management in Irish prehospital care; this would be best achieved by the establishment of a prehospital airway registry.

\section{Limitations}

As a cross-sectional study that uses a self-administered questionnaire, self-reporting bias (both social desirability and recall bias) was possible. As this is a self-reported survey, it has not been possible to verify the results against audit data to check for accuracy. This may lead to reported figures being over or underreported. Therefore, no solid conclusions can be drawn from the reported data without verification. Nor can the data be used to reflect the competence of the respondents based on their answers.

The response rate is $27 \%$ which may not reflect a complete picture of current practice and opinion. We do however believe that the quality improvement implications of our results would be beneficial not only for our study population but also for other countries.

\section{Abbreviations}

ALS: Advanced Life Support

AP: Advanced Paramedic

EMS: Emergency Medical Service

ETI: Endotracheal Intubation

ETT: Endotracheal Tube

EPCR: Electronic Patient Care Report

HALO: High Acuity Low Occurrence

LMA: Laryngeal Mask Airway

NPA: Nasopharyngeal Airway

OPA: Oropharyngeal Airway

QR: Quick Response

\section{Declarations}




\section{Acknowledgment}

The authors would like to acknowledge the survey participants from the National Ambulance Service and Dublin Fire Brigade.

\section{Authors Contributions}

Conception: AH, DH. Survey design, acquisition and analysis: $A H, D H$. Drafted the paper: $A H, D H$. Substantively revised the paper: SM, COD. Approved submitted version: AH, SM, COD, DH.

\section{Funding}

Not applicable

\section{Availability of data}

The survey result dataset is available from the corresponding author on reasonable request.

\section{Ethics approval and consent to participate}

Ethical approval granted by the University Hospital Limerick, Ireland research ethics committee and approval to conduct the survey was granted by the National Ambulance Service research committee.

\section{Consent for publication}

Survey participants were informed prior to completing the survey that the survey results would be published.

\section{Competing interests}

The authors declare that they have no competing interests.

\section{References}

1. Consensus Statement. A framework for safe and effective intubation by paramedics (2018) College of Paramedics

2. Crewdson K, Lockey D, Voelckel W, Temesvari P, Lossius HM (2019) Best Practice advice on prehospital emergency anaesthesia and advanced airway management Scandinavian Journal of trauma Resuscitation and Emergency Medicine; 27, 6

3. Prekker M, Kwok H, Shin J, Carlbom D, Grabinsky A, Rea T (2014) The Process of Prehospital Airway Management: Challenges and Solutions During Paramedic Endotracheal Intubation Critical Care Medicine 42(6): 1372-1378

4. Toda J, Akira A, Arakawa J(2013) Learning Curve for Paramedic Endotracheal Intubation and Complications International Journal of Emergency Medicine 2013, 6:38 
5. Joint Royal Colleges Ambulance Liaison Committee (2008) A Critical Reassessment of Ambulance Service Airway Management in Prehospital Care

6. DysonK, Bray JE, Smith K, Bernard S, Straney L, Nair R, Finn J (2017) Paramedic Intubation Experience Is Associated with Successful Tube Placement but Not Cardiac Arrest Survival Annals of Emergency Medicine Sep;70(3):382-390.e1

7. Ono Y, Tanigawa K,Kakamu T, Shinohara K, Iseki K (2018) Out-of hospital endotracheal intubation experience, confidence and confidence-associated factors among Northern Japanese emergency lifesaving technicians: a population-based cross-sectional study. BMJ Open 2018021858

8. Crewdson K, Lockey J, RØislien, Lossius HM, Rehn M (2017) The Success of prehospital tracheal intubation by different pre-hospital providers: a systematic literature review and meta analysis Critical Care 21, 31

9. McQueen C, Crombie N, Hulme J, Cormack S, Hussain N, Ludwig F, Wheaton S (2015) Prehospital anaesthesia performed by physician/critical care paramedic teams in a major trauma network in the UK: a 12 month review of practice Emergency Medicine Journal 32(1):65-9

10. Central Statistics Office of Ireland (2016) Census 2016

11. Clinical Practice Guidelines - Advanced Paramedic (2017) Pre Hospital Emergency Care Council

12. Sunde, G.A., Kottmann, A., Heltne, J.K. Sandberg M., Gellerfors M., Kruger A., Lockey D., Sollid J.M. (2018) Standardised data reporting from pre-hospital advanced airway management - a nominal group technique update of the Utstein-style airway template. Scand J Trauma Resusc Emerg Med 26, 46

13. Kovacs G, Bullock G, Ackroyd-Stolarz S, Cain E, Petrie D (2000) A Randomized Controlled Trial on the Effect of Educational Interventions in Promoting Airway Management Skill Maintenance Annals of Emergency Medicine Oct;36(4):301-9

14. Trimmel H, Beywinkler C, Hornung S, Kreutziger J, Voelcke WG (2017) In-Hospital Airway Management Training for Non-Anaesthesiologist EMS Physicians: A Descriptive Quality Control Study Scandinavian Journal of Trauma, Resuscitation and Emergency Medicine 25:45

15. Knox S, Cullen W, Collins N, Dunne C. (2013) First evaluation of CPD advanced paramedic teaching methods in Ireland. Journal of Paramedic Practice 5:29-35

16. Maloney S, Storr M, Morgan P, Illic D (2013) The effect of student self-video of performance on clinical skill competency: a randomised controlled trial Advances in Health Science Education 18:8189

\section{Figures}




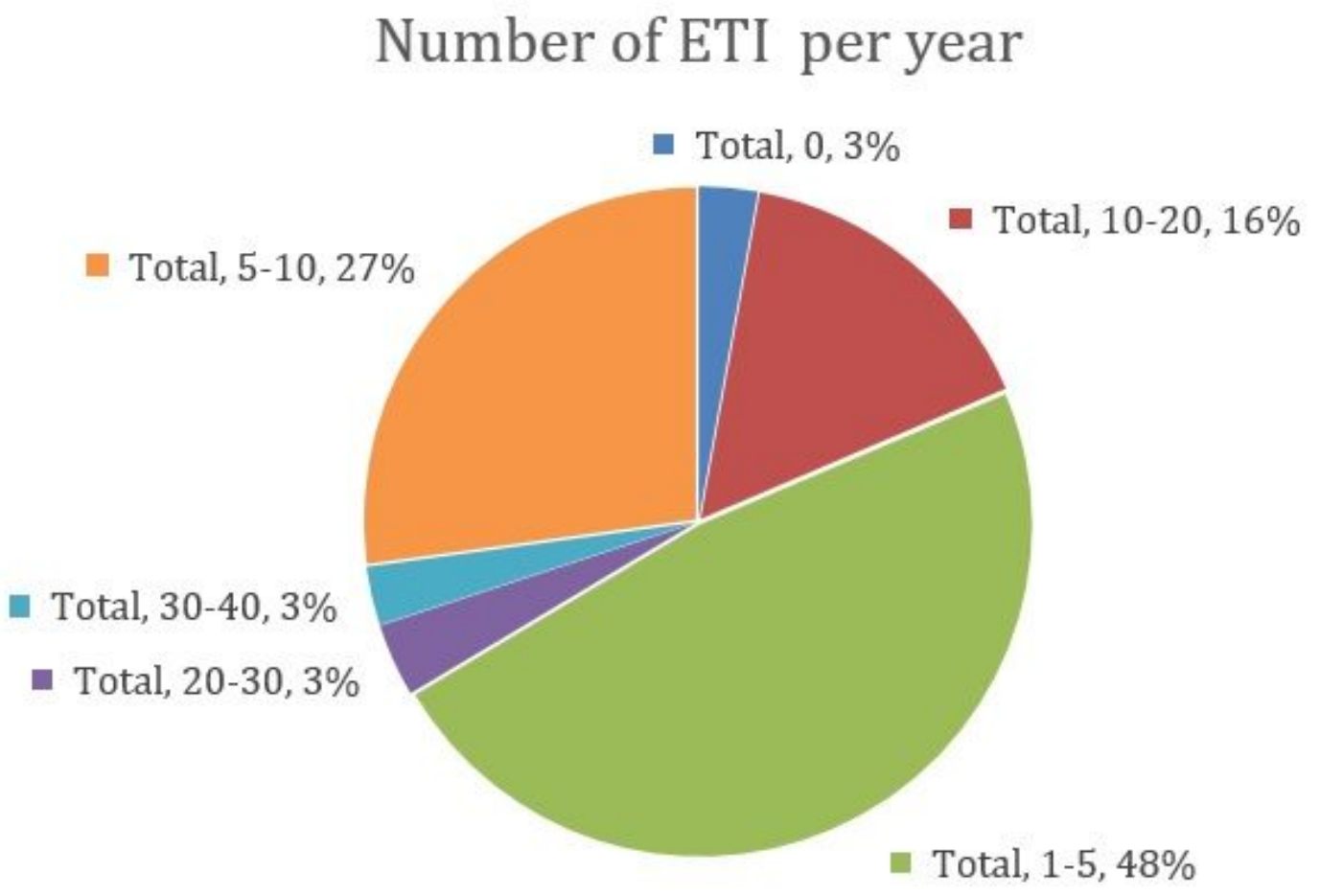

Figure 1

Annual ETI total by AP

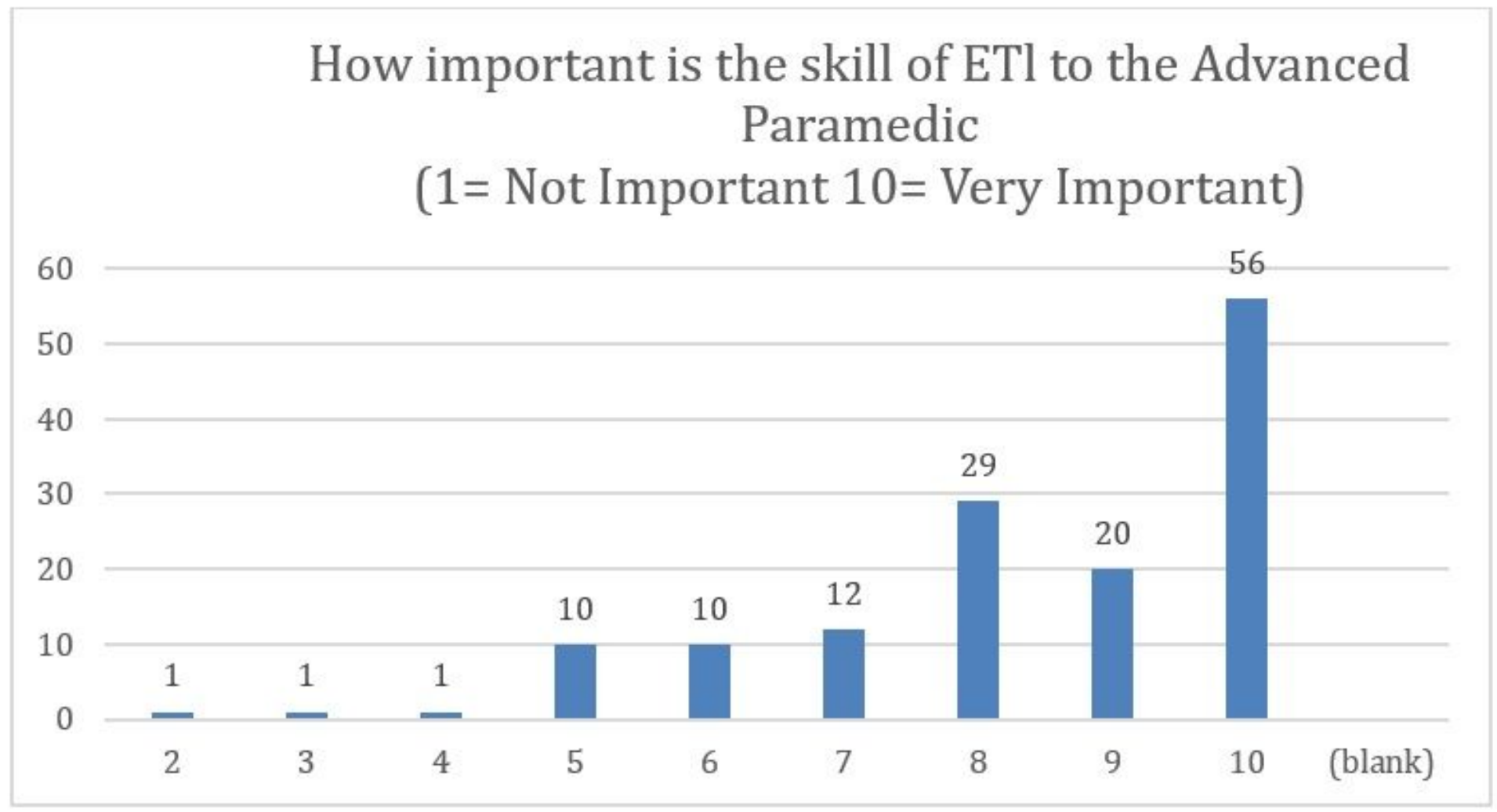

Figure 2 
Importance of ETI by AP

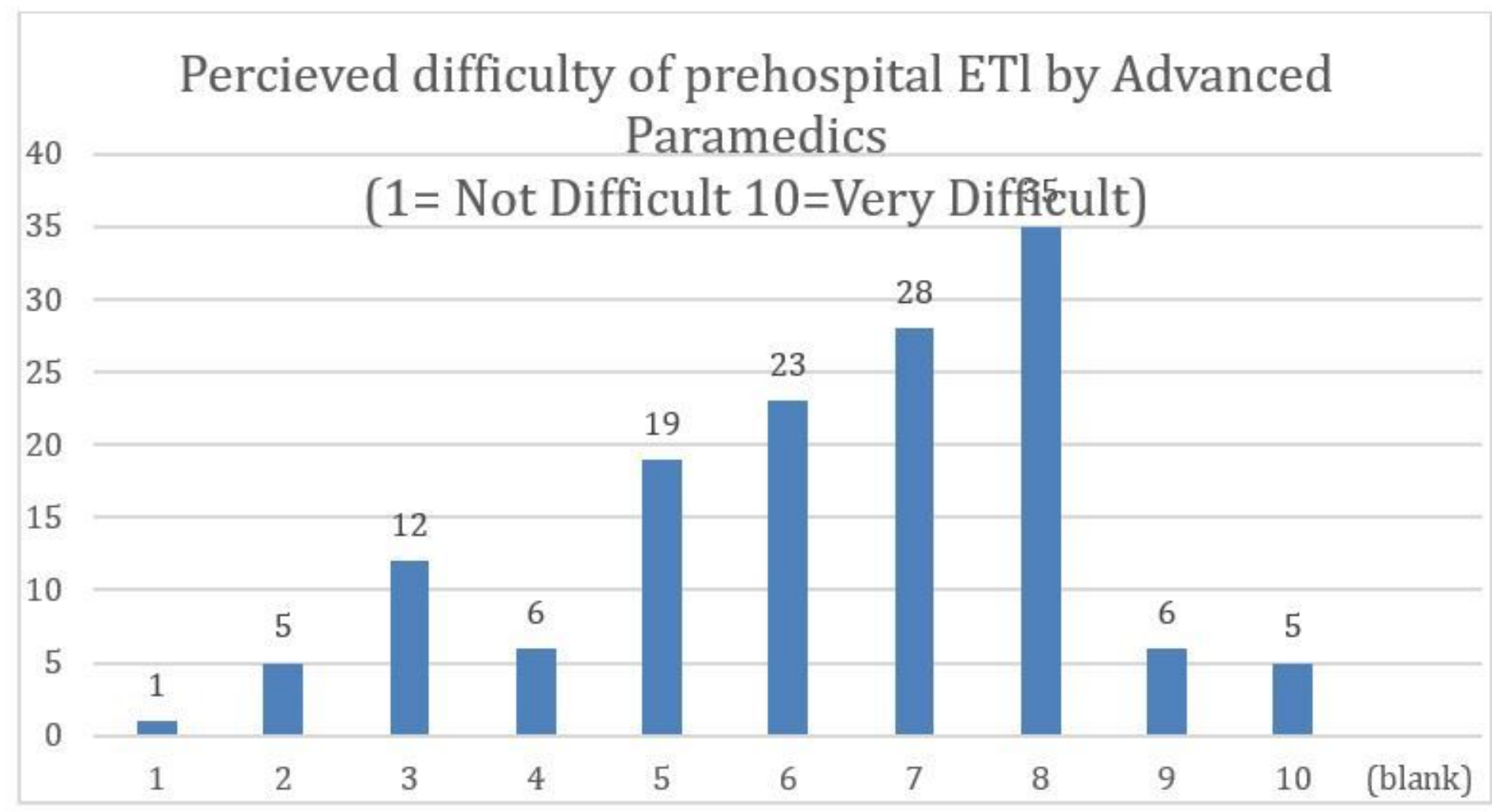

Figure 3

Difficulty of ETI by AP

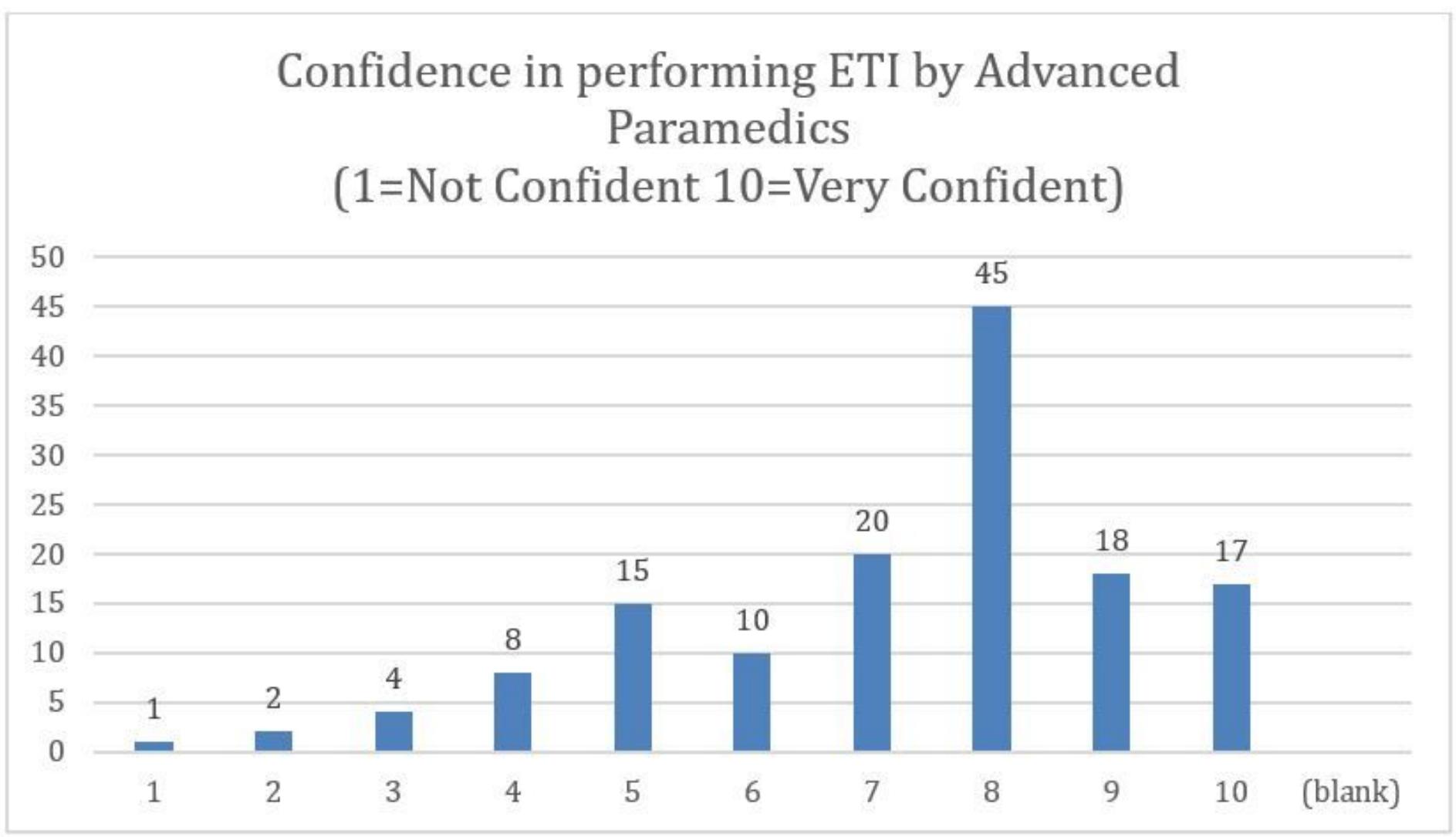


Figure 4

Confidence of ETI performance by AP

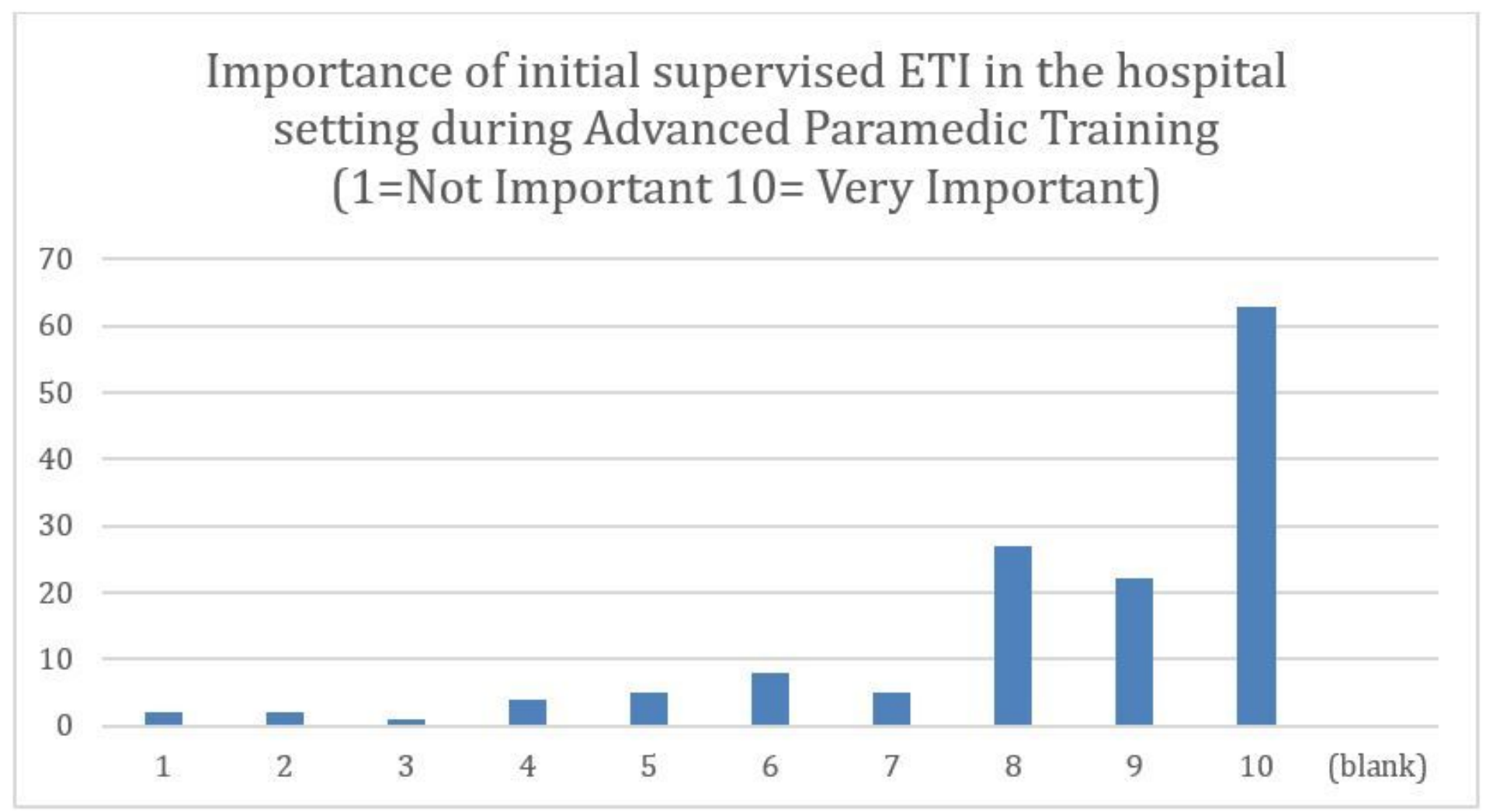

\section{Figure 5}

Importance of supervised in hospital ETI training for AP 


\section{Advanced Paramedic perception of ongoing CPC / experience to maintain ETI competency (1=Not Adequate 10=Very Adequete)}

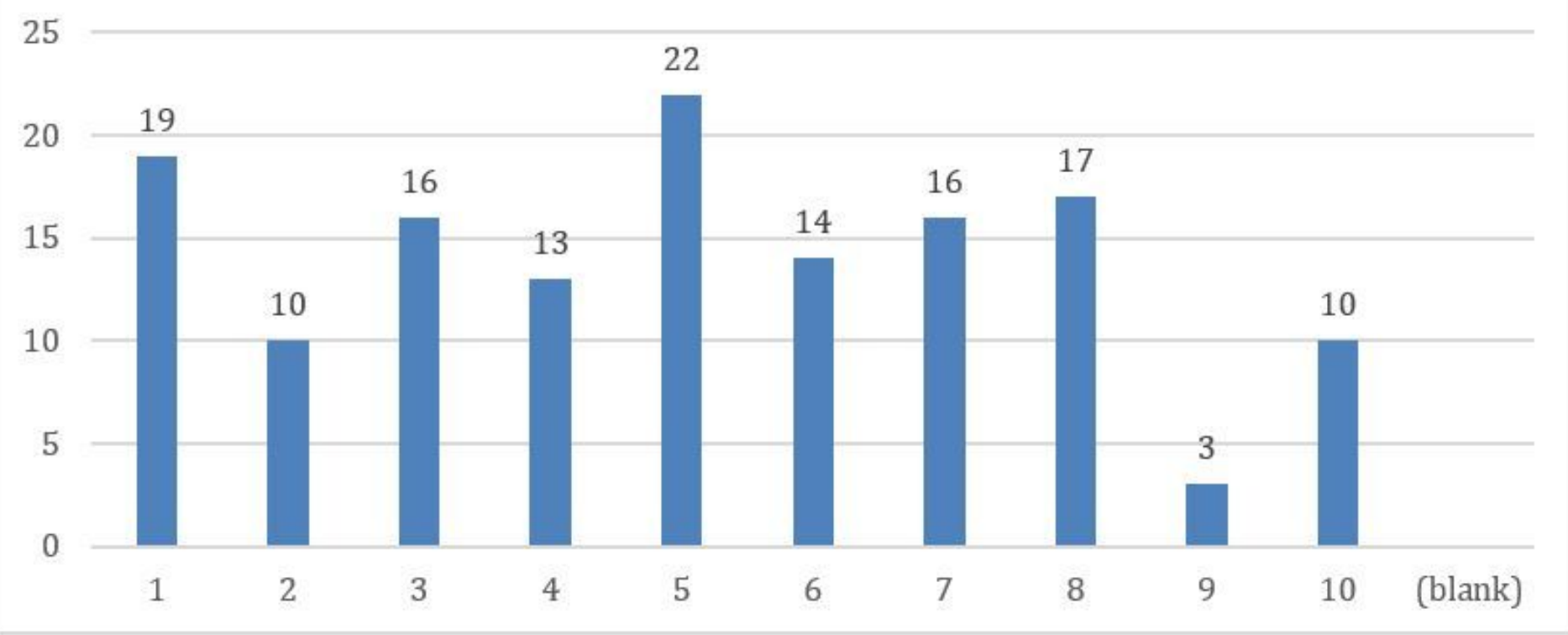

\section{Figure 6}

Perception of current competency assurance for ETI by AP

\section{Advanced Paramedic suggestions for ETI competency assurance}

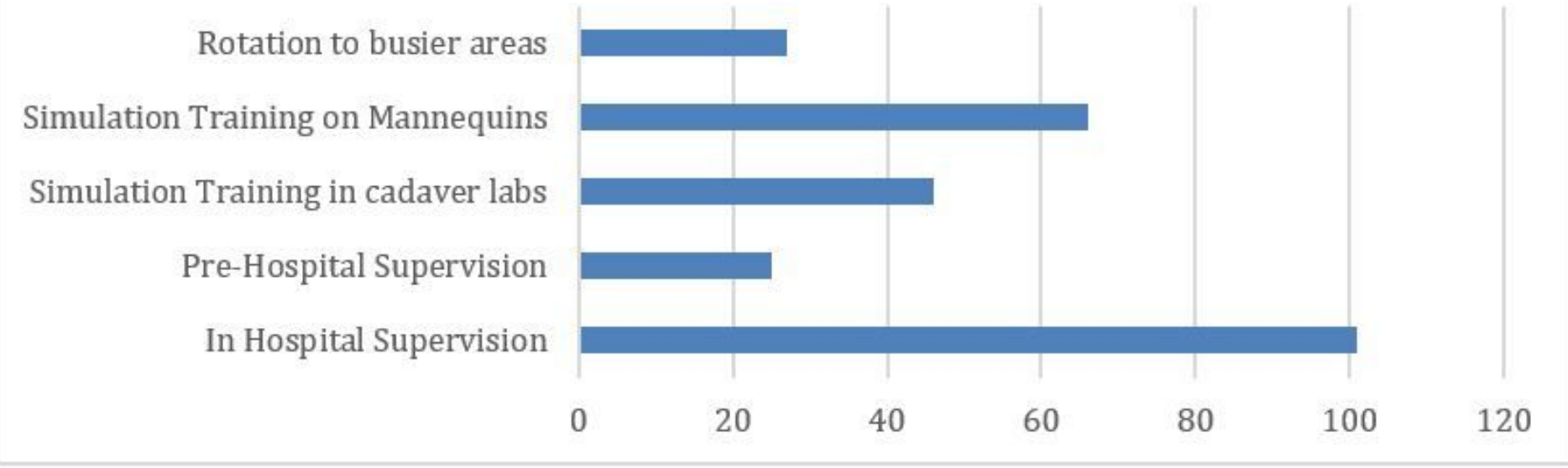

\section{Figure 7}

Suggestion of competency assurance for ETI by A 